\title{
MedienPädagogik
}

www. medienpaed.com

Zeitschrift für

Theorie und Praxis

der Medienbildung

ISSN 1424-3636

Themenheft Nr. 23: Visuelle Kompetenz

\section{Fotografische Wirklichkeitskonstruktion im Spannungsfeld von Bildgestaltung und Bildmanipulation}

Peter Holzwarth

\begin{abstract}
Der folgende Beitrag stellt die Frage nach den Grenzen zwischen Bildgestaltung und Bildmanipulation und gibt einen Überblick zu verschiedenen gestalterischen Strategien, durch die Bedeutungen konstruiert bzw. manipuliert werden können. Am Ende werden Potenziale für pädagogische Kontexte diskutiert.
\end{abstract}

Das Zeitalter der paradoxen Logik des Bildes ist das Zeitalter der grenzenlosen Bildmanipulation, das Zeitalter des manipulativen Einsatzes von Bildern. Bilder unterschiedlicher Herkunft können miteinander vermischt werden, jeder Bildpunkt ist der Manipulation zugänglich, Pixel für Pixel. (Bühl 1996, 190)

Calvin: "This is what I like about photography. People think cameras always tell the truth. They think the camera is a dispassionate machine that records only facts, but really, cameras lie all the time! Select the facts and you manipulate the truth! For example, I've cleared off this corner of my bed. Take a picture of me here, but crop out all the mess around me, so it looks like I keep my room tidy.» (Calvin \& Hobbes Comic, Bill Watterson 1993, 148)

\section{Einleitung}

Wie jede Erfindung hat auch die Fotografie ambivalente Veränderungen mit sich gebracht. Sie hat einerseits geholfen, die Welt anders zu sehen und besser zu verstehen, andererseits wirft sie immer wieder neue Fragen auf. Schon seit langem stellt sich etwa bei einem Foto die Frage, ob eine so vorgefundene Situation dokumentiert wurde oder ob es sich vielmehr um eine Inszenierung handelt und das Dargestellte vor der Aufnahme bewusst arrangiert wurde. Historisch jünger ist die Frage, ob ein Foto mit Techniken der digitalen Nachproduktion bearbeitet wurde. Beide Fragen spielen auch für die Entwicklung von visueller Kompetenz eine zentrale Rolle. 


\section{Fotografie, Wirklichkeitskonstruktion und Erinnerung}

Ein grosser Teil des Reizes, der von der fotografischen Darstellung ausgeht, besteht wohl darin, dass der entsprechende Wirklichkeitsausschnitt einerseits in einer gewissen Weise objektiv abgebildet wird, andererseits aber dabei auch subjektive Perspektiven deutlich werden. Das fotografierende Individuum wählt das Objekt, den Ausschnitt und die Einstellungen der Kamera, doch darüber hinaus fliesst keine Subjektivität in den Abbildungsprozess ein. Der Fotoapparat unterscheidet nicht zwischen Wichtigem und Unwichtigem (Buddemeier 1981, 15). In gewisser Hinsicht handelt es sich um eine getreue Abbildung der sichtbaren Wirklichkeit, bei der die Objekte scheinbar so abgebildet werden, wie sie wirklich sind. Barthes betont, es liesse sich angesichts eines abgebildeten Objektes nicht leugnen, dass die Sache dagewesen ist und spricht daher von einem «Es-ist-so-gewesen» der Fotografie (Barthes 1989, 87).?

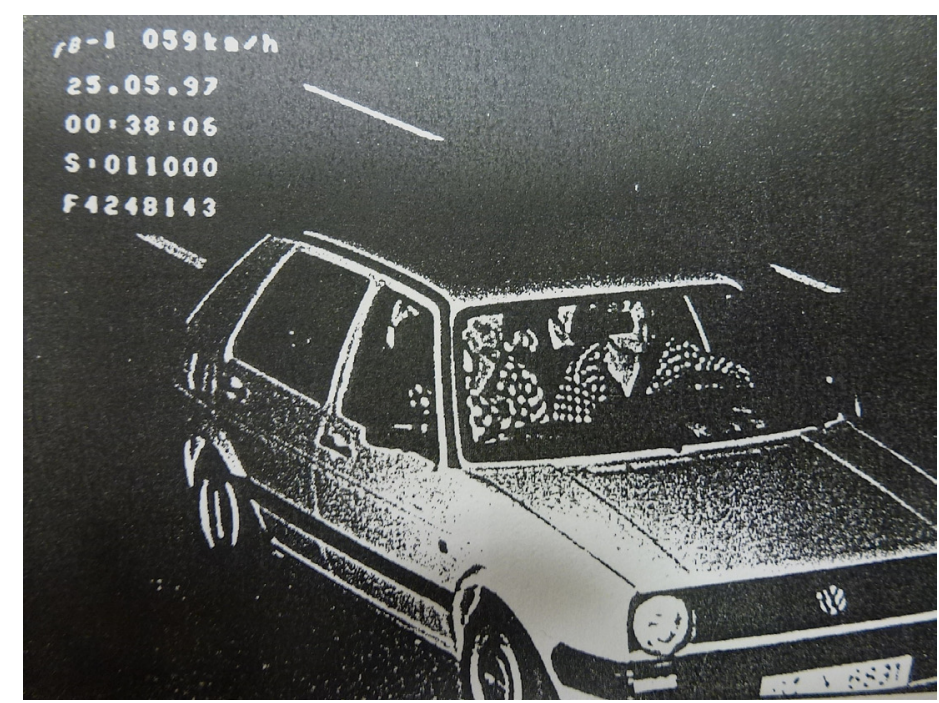

Abb. 1: «Es-ist-so-gewesen» (Barthes 1989, 87) - Radarfoto als Beweis

Neben der Objektivität der Abbildung ist es auch die Vorstellung einer Konservierung über die Zeit hinweg, welche das Fotografieren so anziehend erscheinen lässt. In Anlehnung an Bourdieu weist Schneider darauf hin, dass die materielle Konsistenz eines Fotos «als Schutz gegen die Vergänglichkeit der Zeit fungiert»

1 Ähnlich argumentiert Susan Sontag:

«Eine Fotografie gilt als unwiderleglicher Beweis dafür, dass ein bestimmtes Ereignis sich tatsächlich so abgespielt hat. Das Bild mag verzerren; immer aber besteht Grund zu der Annahme, dass etwas existiert - oder existiert hat -, das dem gleicht, was auf dem Bild zu sehen ist» (Sontag 1995, $11 \mathrm{f}$.$) .$ 
(Schneider 1993, 449). Neben der gedächtnismässigen Erinnerung besteht durch die Fotografie die Möglichkeit, flüchtige Aspekte der Wirklichkeit bildlich festzuhalten und diese unter relativer Unabhängigkeit von Raum und Zeit weiten Personenkreisen zugänglich zu machen. Die Fotografie ermöglicht beispielsweise den Zugriff auf visuelle Informationen über das Leben in vergangenen Zeiten und an anderen Orten der Welt. Es ist jedoch zu betonen, dass einer Informationsvermittlung über das Medium Bild eine andere Qualität innewohnt als der Informationsvermittlung, welche mit der direkten Erfahrung (Primärerfahrung) einhergeht. Viele visuelle Informationen haben die Eigenschaft, dass sie sich der direkten empirischen Nachprüfung entziehen, weil der abgebildete Sachverhalt einem vergangenen Zeitpunkt angehört oder aber sich an einem anderen Ort vollzogen hat. Die vielfach konstatierte Objektivität der Fotografie muss daher als ein radikal ambivalentes und kontextspezifisches Phänomen betrachtet werden. In Umschreibung von Barthes' Diktum könnte man sagen: "Manchmal ist es unabhängig vom fotografierenden Subjekt so gewesen, manchmal ist es nur durch die Inszenierung so gewesen, manchmal ist es nicht so gewesen.» Oder: «Es könnte so gewesen sein.» Sowohl durch Inszenierung als auch durch selektive Auswahl dessen, was abgebildet werden soll, kann eine antizipierende Produktion von Erinnerung und Bedeutung vorgenommen werden. Dieser Gedanke wird in einem Dialog aus Wattersons populärer Comic-Serie «Calvin and Hobbes» schön veranschaulicht:

Calvin: «OK, there's a picture of me looking well-adjusted and playing sports. That ought to do it.»

Hobbes: «You hate sports.»

Calvin: «Yeah, but people believe what they see, and now we've got a photographic document of a fake childhood ready for any future biographical needs I may have!» (Watterson 1993, 149)

Oder um noch ein zweites Beispiel anzuführen: Jack Kerouac verweist in seinem Roman "On the Road» auf die mögliche Differenz von Lebensgefühlen zum Zeitpunkt der fotografischen Aufnahmen und den später zugeschriebenen Bedeutungen:

«Dean took out other pictures. I realized these were all the snapshots which our children would look at someday with wonder, thinking their parents had lived smooth, well-ordered, stabilized-within-the-photo lives and got up in the morning to walk proudly on the sidewalks of life, never dreaming the raggedy madness and riot of our actual lives, our actual night, the hell of it, the senseless nightmare road. All of it inside endless and beginning less emptiness. Pitiful forms of ignorance.» (Kerouac 1991, 254) 
Es ist möglich, die Dinge (und auch sich selbst) so abzubilden, wie man es in Erinnerung behalten möchte. Im Rahmen eines Symposiums der Deutschen Gesellschaft für Photographie («Kunst und Kommerz - Perspektiven in der Photographie») in der Staatlichen Akademie der bildenden Künste Stuttgart am 20. April 1996 äusserte der amerikanische Fotograf Duane Michals den Satz: «Past is what you choose to remember.» Damit brachte er zum Ausdruck, dass das Individuum aktiv und selektiv an der Rekonstruktion der Vergangenheit beteiligt ist und damit die eigene Wirklichkeit mitkonstruiert.

Doch nicht nur in der Biographie von Einzelpersonen kann die Fotografie Wirklichkeit produzieren, auch die Weltgeschichte kann mit dem Mittel der Fotografie manipuliert werden. In George Orwells Roman «1984» wird etwa deutlich, wie durch die Kontrolle dessen, was als geschehen angesehen wird, das Bewusstsein und das Verhalten der Menschen gesteuert wird. Mit der Kontrolle über die Vergangenheit wird auch bestimmt, was als wirklich erachtet wird:

«Zwar gab es keinen Menschen dieses Namens auf der Welt, aber ein paar gedruckte Zeilen und zwei gefälschte Photographien würden ihn schnell und ohne grosse Mühe ins Leben rufen.» (Orwell 1990, 45)

Wann hört Bildgestaltung auf und wo beginnt Manipulation? Dieser Frage soll im Folgenden nachgegangen werden.

\section{Bildgestaltung - Bildmanipulation?}

Im Zusammenhang der bewusst eingesetzten Formen fotografischer Wirklichkeitskonstruktion werden unterschiedliche Begriffe verwendet. Man spricht so etwa von Bildgestaltung, Bildbearbeitung, Bildcollage, Fotomontage, Bildretusche, Fotomanipulation oder Bildmanipulation. Diese Begriffe unterscheiden sich in Bezug auf das, was man «ethische Neutralität» hinsichtlich der Fotografie und dem Umgang mit ihr nennen könnte. «Bildmanipulation» und «Fotomanipulation» implizieren eine Täuschungsabsicht, bei «Bildgestaltung» und «Bildbearbeitung» dagegen schwingt keine negative Bedeutung mit. «Bildgestaltung» kann sich weiterhin zeitlich auf alle Phasen der Produktion beziehen, die anderen Begriffe beschreiben hingegen tendenziell eher Prozesse nach der Aufnahme. Wie bereits das erste Motto des Textes deutlich macht, kann zwischen Manipulation am Bild und einem manipulativen Einsatz von Bildern unterschieden werden. Man kann also Fotos manipulieren, ohne sie dann manipulativ einsetzen zu wollen, umgekehrt können aber auch nicht manipulierte Fotos mit einer manipulativen Intention verwendet werden. Tabelle 1 veranschaulicht die beiden Dimensionen anhand von Beispielen (Abb. 2-5): 
Tabelle 1: Dimensionen von Bildmanipulation

\begin{tabular}{|l|l|l|}
\hline & Bildgebrauch mit Manipulationsintention & Bildgebrauch ohne Manipulationsintention \\
\hline $\begin{array}{l}\text { Manipulierte } \\
\text { Bilder }\end{array}$ & $\begin{array}{l}\text { Ein Mann, der politisch in Ungnade ge- } \\
\text { fallen ist, wird aus einem Foto heraus } \\
\text { retuschiert und den Betrachtenden wird } \\
\text { suggeriert, dass es ihn in diesem Kontext } \\
\text { nicht gegeben hat. }\end{array}$ & $\begin{array}{l}\text { Ein Foto, das einen Froschkörper mit } \\
\text { Schweinekopf zeigt, wird im Internet veröf- } \\
\text { fentlicht. }\end{array}$ \\
\hline $\begin{array}{l}\text { Nicht } \\
\text { manipulierte } \\
\text { Bilder }\end{array}$ & $\begin{array}{l}\text { Um ein positives Bild von einem Krieg zu } \\
\text { etablieren werden bewusst nicht Fotos } \\
\text { von Verletzten und Toten gezeigt, son- } \\
\text { dern solche, die Soldaten beim gemütli- } \\
\text { chen Zusammensein zeigen. }\end{array}$ & Ein Mann zeigt Freunden Urlaubsbilder aus \\
\hline
\end{tabular}
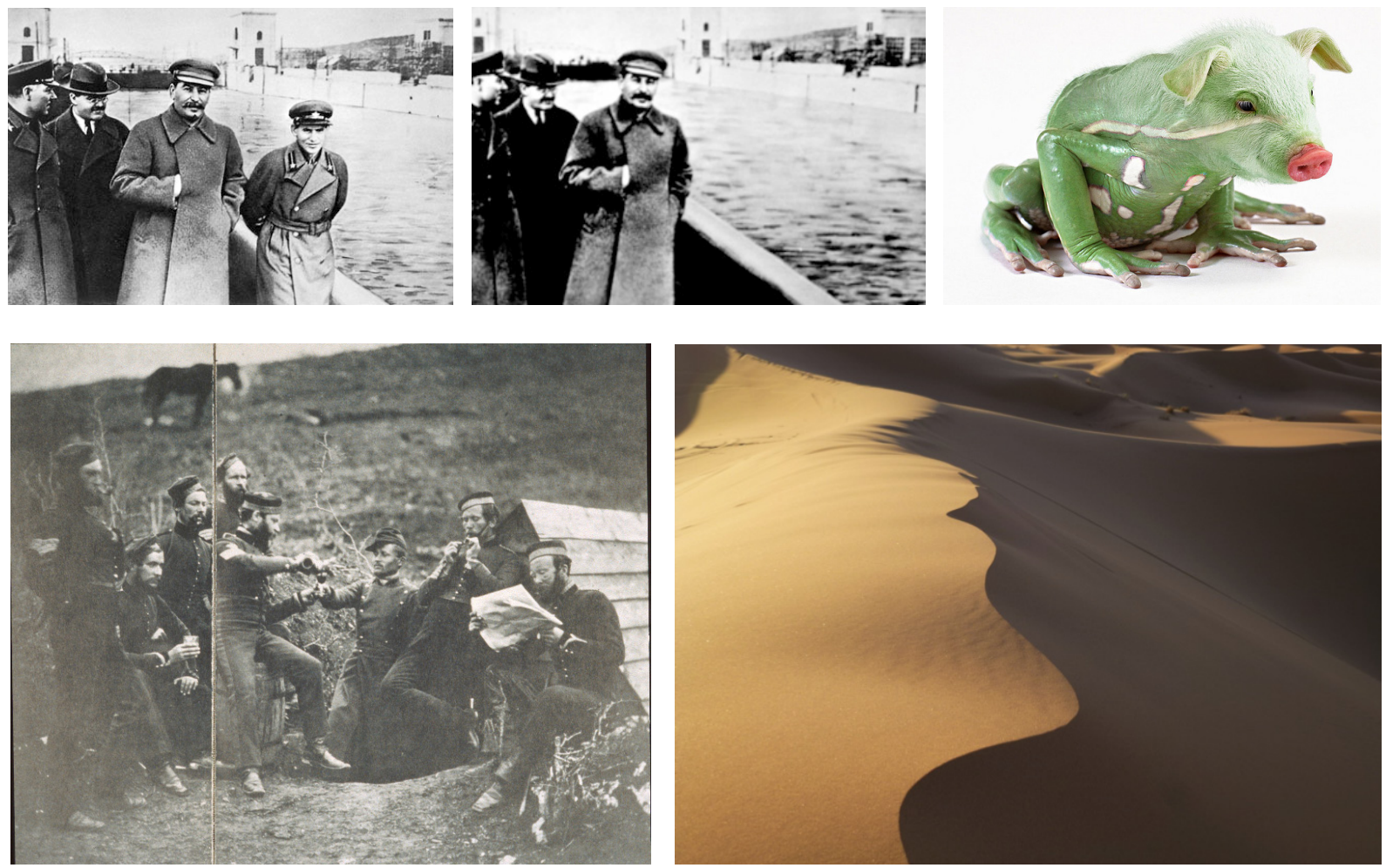

Abb. 2-5: Bildbeispiele zu Tabelle 1

2 Haus der Geschichte der Bundesrepublik Deutschland 2003, 84 oder http://en.wikipedia.org/wiki/ Photo_manipulation.

3 http://www.flickr.com/photos/42311564@N00/494023970/in/set-72157601948950307.

4 Vgl. dazu Haus der Geschichte der Bundesrepublik Deutschland 2003, 16; http://www.Isg.musin. de/geschichte/Material/referate/lkg/krimkrieg_1856.htm [letzter Zugriff auf diese und alle weiteren Webseiten am 9.1.2013]. 
Die Grenzen zwischen «Bildgestaltung» und «Bildmanipulation» können dabei fliessend sein. Zeitlich gesehen können mögliche Bedeutungspotenziale eines Fotos vor, während oder nach der Aufnahme geprägt bzw. verändert werden (vgl. Holzwarth 2012). Dazu eine Beispielserie (Tabelle 2):

Tabelle 2: Zeitpunkt der Bildmanipulation im Verhältnis zur Aufnahme

\begin{tabular}{|l|l|l|}
\hline \multicolumn{2}{|l|}{ Bedeutungsveränderung/Manipulation der Aufnahme (Bildwirklichkeiten inszenieren) } \\
\hline vor & während & nach \\
\hline $\begin{array}{l}\text { Eine Flasche vom Strand vor dem } \\
\text { Fotografieren entfernen }\end{array}$ & $\begin{array}{l}\text { Den Strand so fotografieren, dass } \\
\text { sich die Flasche ausserhalb des } \\
\text { gewählten Bildausschnitts befin- } \\
\text { det }\end{array}$ & $\begin{array}{l}\text { Die Flasche mit Hilfe digitaler } \\
\text { Bildbearbeitung aus dem Foto } \\
\text { entfernen }\end{array}$ \\
\hline
\end{tabular}

Streng genommen könnte bei jedem Foto von Manipulation gesprochen werden, da notwendigerweise die Cadrierung Teil des fotografischen Akts ist und so nur ein Teil der Wirklichkeit gewählt werden kann, andere hingehen für die Betrachter ausgeblendet werden. Eine solch allgemeine Bestimmung von Manipulation wäre jedoch nicht sehr trennscharf. Es ist sinnvoller, nur dann von einer Bildmanipulation zu sprechen, wenn die bewusste Intention vorliegt, Menschen gegen ihren Willen zu beeinflussen. Bei der Frage nach Manipulation spielt also der Verwendungskontext eine entscheidende Rolle. Durchaus in diesem Sinne wird in einem medienpädagogischen Dossier über Bildmanipulation folgende Definition vorgeschlagen:

Von Manipulation (im engeren Sinn) sollte nur dann gesprochen werden, wenn Bilder eingesetzt werden, um eine bestimmte Wirklichkeit vorzutäuschen und die Betrachter vorsätzlich und zum eigenen Vorteil zu beeinflussen, z. B. zum Kauf von Produkten, zur Erhöhung einer Attraktivitätseinschätzung, zur Übernahme einer Meinung oder zur Wahl eines Politikers. (Holzwarth 2012, 7)

«Manipulation» impliziert oft die Vorstellung einer unbewussten Wirkung von Bildern, der der Mensch passiv ausgeliefert ist. Viele Autorinnen und Autoren betonen jedoch, dass Menschen sich Medien aktiv, selektiv und kontextabhängig aneignen (vgl. Uses-and-Gratification-Ansatz, Dynamisch-transaktionaler Ansatz: Sander, von Gross u. Hugger 2008 173-184; Encoding-Decoding-Modell: Hepp 1999, 110-118). Generell sind beide Perspektiven wichtig: «Was machen die Medien mit den Menschen?» und «Was machen die Menschen mit den Medien?». Es 
stellt sich die Frage, ob das Konzept «Manipulation» noch vereinbar ist mit einer modernen Vorstellung von Medienaneignung.

\section{Möglichkeiten der Bedeutungskonstruktion}

Folgende Strategien können Bedeutungen konstruieren bzw. verändern und mit einer manipulativen Absicht verbunden sein (Tabelle 3):

Tabelle 3: Bedeutungskonstruktionen im Verhältnis zum Zeitpunkt der Aufnahme

\begin{tabular}{|c|c|c|}
\hline Vor dem Fotografieren & Während des Fotografierens & Nach dem Fotografieren \\
\hline - Inszenierung & $\begin{array}{l}\text { - Einstellungsgrösse / Ausschnitt } \\
\text { - Perspektive } \\
\text { - Schärfe } \\
\text { - Objektivwahl }\end{array}$ & $\begin{array}{l}\text { - Ausschnitt } \\
\text { - Farbe } \\
\text { - Bildrotation } \\
\text { - Digitale Fotomontage «Copy } \\
\text { - and Paste» / Collage } \\
\text { - Präsentationskontext } \\
\text { - Text } \\
\text { - Bildnachbarschaft } \\
\text { - Musik und Ton } \\
\text { - Auswahl eines Einzelfotos } \\
\text { - Schärfe }\end{array}$ \\
\hline
\end{tabular}

\section{Bedeutungskonstruktion durch Inszenierung}

Viele fotografische Abbildungen haben die Eigenschaft, dass man ihnen nicht ansehen kann, ob die auf ihnen abgebildeten Aspekte der Wirklichkeit zuvor extra für die Abbildung inszeniert ("gestellt») wurden, oder ob ein Wirklichkeitsausschnitt abgebildet wurde, ohne vorher arrangiert worden zu sein. Bei manchen fotografischen Genres wie z. B. der traditionellen Werbefotografie und manchen Spielarten künstlerischer Fotografie scheint es a priori evident zu sein, dass das Abgebildete eine Inszenierung erfahren hat, bei anderen Bereichen dagegen (z. B. der dokumentarischen oder auch der Pressefotografie) wird eher von einer Authentizität der Bilder ausgegangen. Es kann auch Abbildungen geben, die augenscheinlich authentisch wirken, deren scheinbare Authentizität aber intendiert ist: "Gerade ganz berühmte Reportagebilder sind manchmal - wie sich später herausstellt keine Momentaufnahmen, sondern im nachhinein gestellt» (Schuster 1996, 201). Ein bekanntes Beispiel stellt die Aufnahme des russischen Fotografen Chaldejs vom Hissen der Fahne auf dem Berliner Reichstag 1945 dar:

Der Fotograf sucht eine optimale Komposition, einen beeindruckenden Hintergrund für sein Bild - und findet ihn: die Ostseite des Reichstags, Richtung Brandenburger Tor. Die Ruinen des zerbombten Berlin sind von hier 
aus deutlich zu sehen. [...] Eine Serie von Bildern entsteht. [...] Die Agentur beabsichtigt, es als offizielles Foto zu verbreiten. Bedingung hierfür ist jedoch, dass Chaldej eine Korrektur vornimmt. Der sowjetische Offizier, der seinen Kameraden auf der Säule stützt, trägt - deutlich zu sehen - an jedem Handgelenk eine Armbanduhr. Plünderung darf auf einem offiziellen Foto jedoch nicht dokumentiert sein. Chaldej muss eine Uhr entfernen. Dieses Foto, das im Lauf der Zeit zu einer Ikone wird, ist also nicht nur kunstvoll inszeniert, sondern zudem retouchiert. (Haus der Geschichte der Bundesrepublik Deutschland 2003, 45 u. 47) $)^{5}$

\section{Bedeutungskonstruktion durch die Wahl der Einstellungsgrösse bzw. des Ausschnitts}

Zeitlich gesehen kann die Ausschnittwahl während des Fotografierens erfolgen oder aber im Nachhinein, wenn aus einem bereits bestehenden Foto ein kleinerer Ausschnitt gewählt wird.

Jede fotografische Aufnahme bedeutet per se eine Bedeutungsveränderung des Abgebildeten, da dieses aus seinem Kontext herausgelöst wird und damit notwendigerweise eine Entkontextualisierung erfährt. Allein die Tatsache, dass eine Sache fotografisch abgebildet wird, stellt schon eine Bedeutungsveränderung dar: «Fotografieren heisst Bedeutung verleihen» (Sontag 1995, 32).

Mit dieser Entkontextualisierung kann eine Bedeutungsverstärkung oder Bedeutungskonzentration verbunden sein, da die Aufmerksamkeit der Betrachtenden ganz auf die isolierte Figur gerichtet ist und nicht mit dem Kontext geteilt werden muss. Fehlt der natürliche Zusammenhang, der das Objekt normalerweise umgibt, so ermöglichen sich neue Bedeutungsverknüpfungen.

Im zweiten Motto liefert Watterson mit Calvin und Hobbes ein Beispiel für eine durch gezielte Wahl des Ausschnitts erzeugte selektive Bedeutungszuschreibung.

\section{Bedeutungskonstruktion durch Perspektive}

Berets die Wahl der Perspektive entscheidet über mögliche Bedeutungen: Eine Untersicht oder Froschperspektive kann einen Menschen überlegen und mächtig erscheinen lassen, eine Obersicht oder Vogelperspektive dagegen klein und schutzbedürftig (vgl. Holzwarth 2011, 86). In Rap-Videos findet man oft die Macht verleihende Froschperspektive, junge Frauen auf Social-Network-Plattformen machen sich hingegen häufig die Wirkung der Vogelperspektive zu Nutze.

\section{Bedeutungskonstruktion durch Auswahl des Schärfebereichs}

Bei gleich bleibender Perspektive und konstantem Bildausschnitt kann je nach Wahl des Schärfebereichs ein anderes Bedeutungspotenzial ermöglicht werden. Die Entscheidung für einen Schärfefocus kann beim Fotografieren selbst getroffen

5 Vgl. auch Stiegler 2009. 
werden (vgl. Abb. 6-9) oder im Nachhinein (vgl. Abb. 10-13). Ein nachträgliches selektives Schärfen ist beispielsweise mit dem Foto-App «Instagram» möglich.
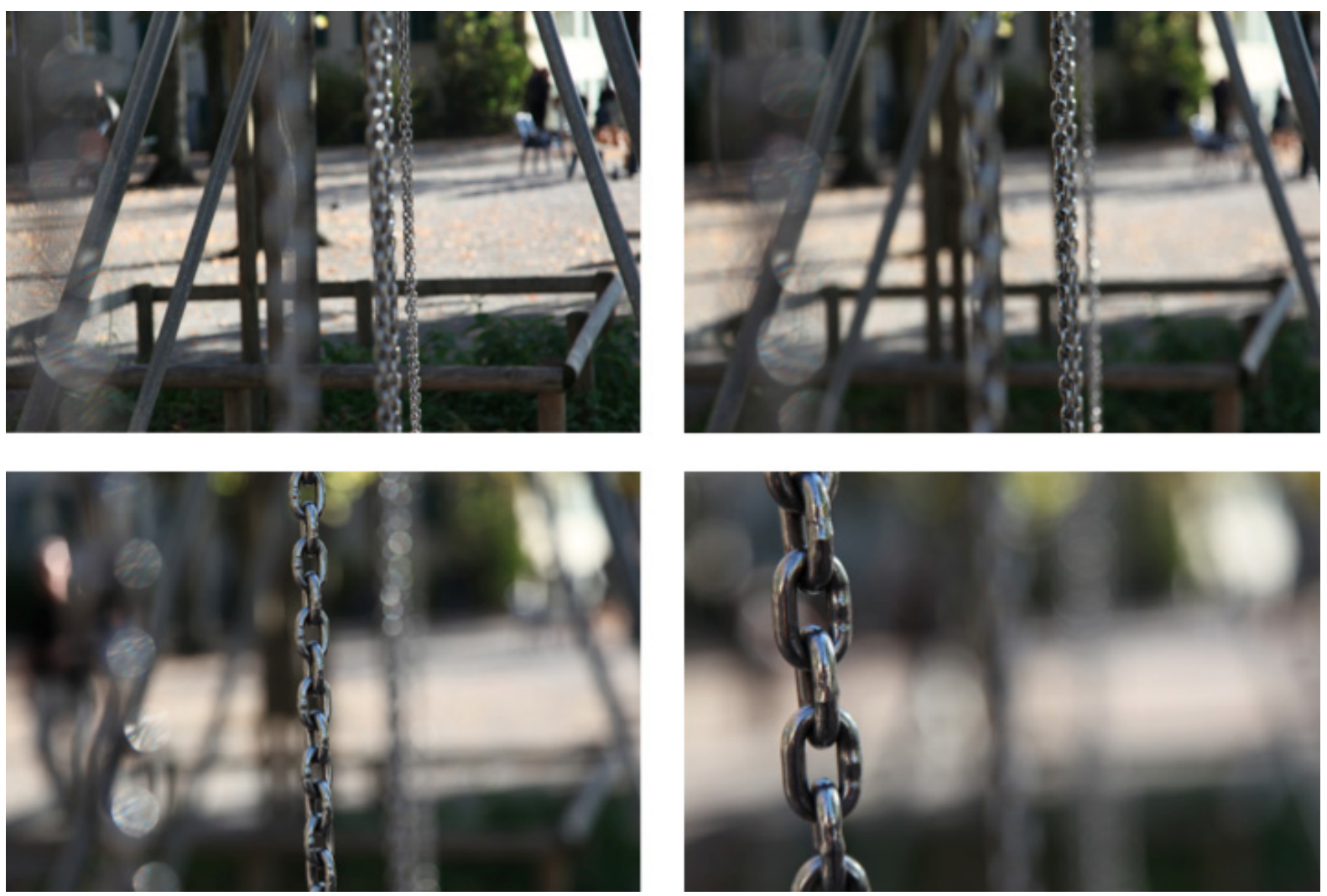

Abb. 6-9: Bedeutungskonstruktion durch Schärfeeinstellung beim Fotografieren: Gleicher Bildausschnitt - verschiedene Schärfebereiche (Peter Holzwarth)

In diesem Sinne könnte man sagen: «Schärfe verleiht Bedeutung». Im Kontext von Film kann durch die bewusste Verlagerung von Schärfe die Aufmerksamkeit der Zuschauer gesteuert werden.

\section{Bedeutungskonstruktion durch Auswahl des Objektivs}

Je nach Objektiv (Weitwinkel oder Tele) kann ein Gesicht unterschiedlich erscheinen. Wie sieht der entsprechende Mensch wirklich aus? Auch Architekturarrangements können je nach Objektiv vorteilhaft oder unvorteilhaft repräsentiert werden. 

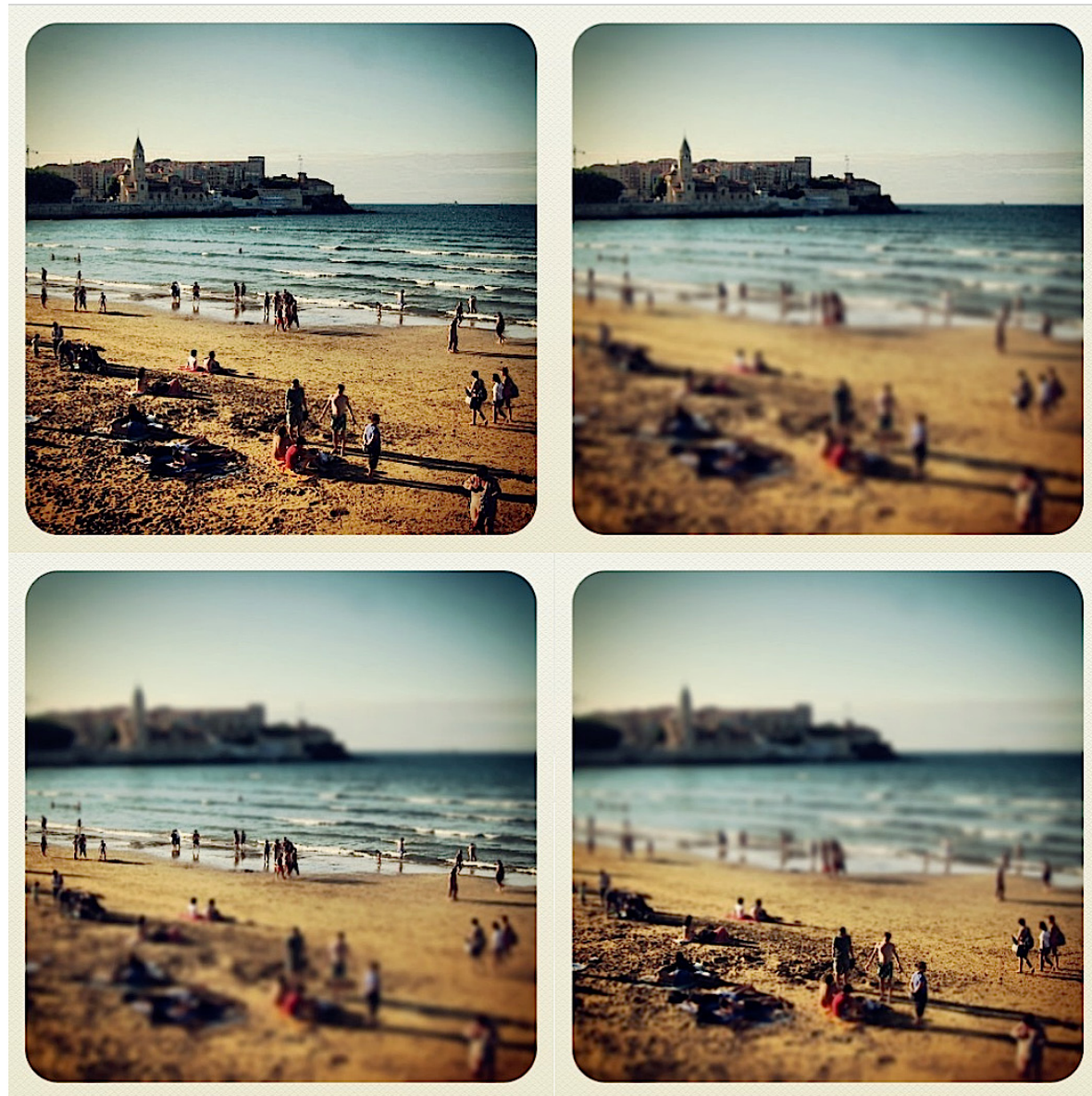

Abb. 10-13: Bedeutungskonstruktion durch Schärfeeinstellung nach dem Fotografieren: Gleiches Foto - verschiedene Schärfebereiche (Peter Holzwarth)

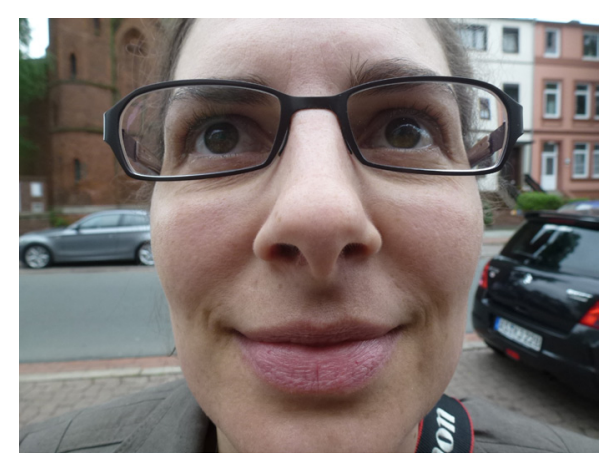

Abb. 14: Weitwinkelaufnahme: Das Gesicht erscheint schmal und sehr plastisch (Peter Holzwarth)

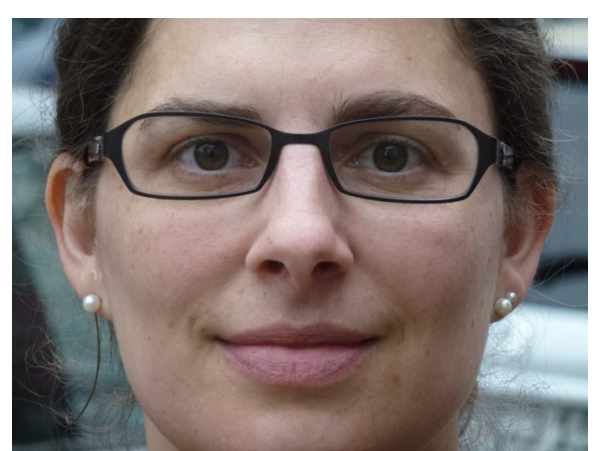

Abb. 15: Teleaufnahme: Das Gesicht erscheint breit und eher flach (Peter Holzwarth) 

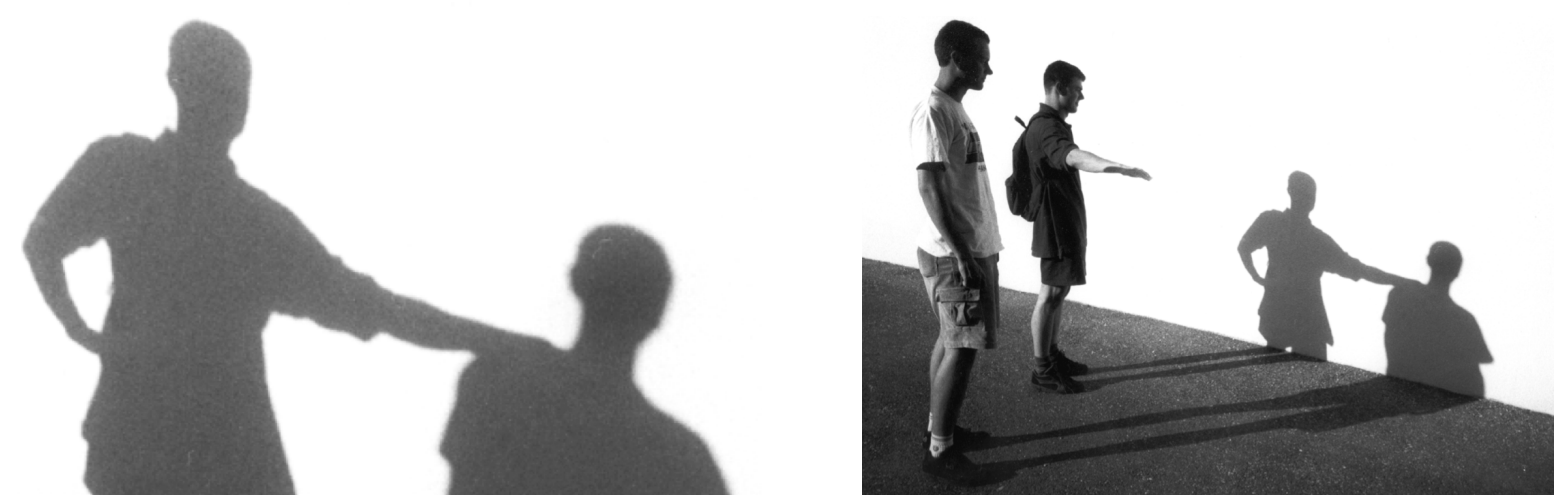

Abb. 16-17: Der Bildausschnitt erzählt eine andere Geschichte als das Gesamtfoto (Peter Holzwarth)

\section{Bedeutungskonstruktion durch die Wahl des Ausschnitts (nach dem Fotografieren)}

Eine Entscheidung für einen bestimmten Wirklichkeitsausschnitt findet bei jedem fotografischen Prozess statt. Eine weitere Ausschnittwahl kann später stattfinden, wenn das Negativ nur zum Teil auf das lichtempfindliche Papier projiziert wird, oder wenn von einem fertigen Bild nur ein Teil genutzt wird. Je nach Fotografie kann die Bedeutung durch einen solchen Ausschnitt extrem verändert werden.

Ein Bildbeispiel von Ursula Dahmen («Der Tagesspiegel») zeigt einen irakischen Soldaten, der von zwei US-Soldaten umringt ist. Je nach Ausschnitt verändert sich die vermeintliche Bedeutung des Bildes dramatisch, denn von der linken Seite ist der Lauf eines Gewehrs auf die Stirn des Soldaten gerichtet, von rechts wird ihm hingegen eine Feldflasche gereicht. Der linke Ausschnitt betont die gewalttätige Seite des Krieges, die rechte Seite dagegen zeigt einen helfenden Aspekt.

\section{Bedeutungskonstruktion durch Farbveränderung}

Die Bedeutung eines Bildes kann auch dadurch verändert werden, dass das ganze Foto oder ein Teil davon anders eingefärbt wird. Ausgelaufenes Wasser kann rot eingefärbt wie ein Blutbad aussehen. ${ }^{6}$ Zu den häufig durchgeführten Farbveränderungen gehören das Umfärben roter Augen und das Umwandeln eines Fotos von Farbe in Schwarz-Weiss. Durch unsere Wahrnehmungsgewohnheiten wird ein Schwarz-Weiss-Foto häufig als künstlerisch, edel oder historisch bedeutsam wahrgenommen.

6 Vgl. die rot eingefärbte Wasserlache vor einem Tempel von Luxor nach dem Attentat vom 17.11.1997: Haus der Geschichte der Bundesrepublik Deutschland 2003, 22; http://www.mfk.ch/ fileadmin/mfkpics/medienbilder/Bilderluegen/LUXOR2.tif. 


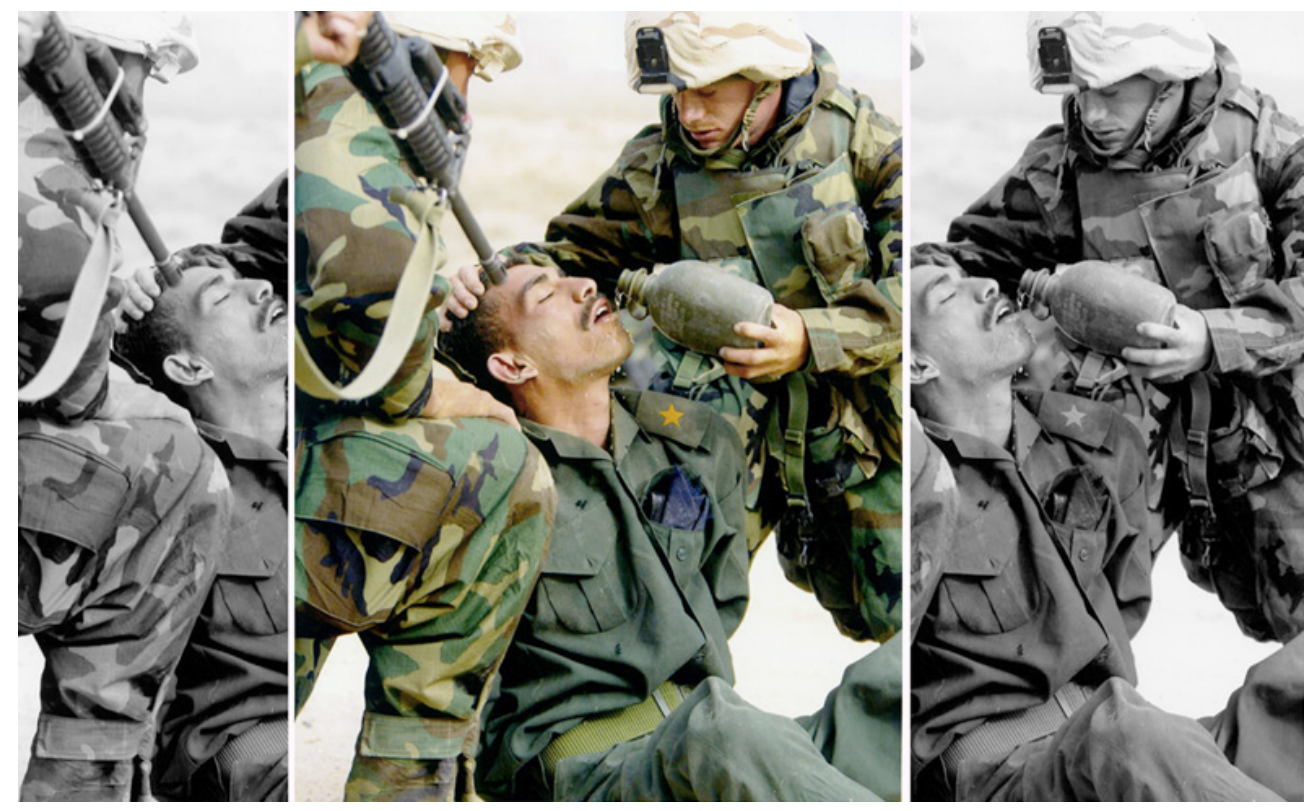

Abb. 18: Je nach Bildausschnitt wird eine andere Haltung gegenüber dem Krieg nahe gelegt (Ursula Dahmen)

\section{Bedeutungskonstruktion durch Bildrotation}

Unter Umständen kann auch durch das Drehen eines Bildes um $90 \mathrm{Grad}$ eine neue Bildbedeutung entstehen. So kann durch simple Rotation aus einer klassischen Situation auf Sportanlässen - Politikerin gratuliert Sportler - suggeriert werden, es habe sich beim Zusammentreffen der beiden Abgebildeten um eine intime Situation gehandelt (vgl. Ammann et al. 2012a, 44; für Abb. siehe Fussnote). ${ }^{7}$

Bedeutungskonstruktion durch digitale Fotomontage ("Copy and Paste» / Collage) Ähnlich wie bei Textverarbeitungsprogrammen kann auch bei digitalen Bildbearbeitungsprogrammen kopiert und eingefügt werden, beispielsweise durch das Hinzufügen einer neuen Bildebene oder mit dem so genannten Kopierstempel. Dies kann unter Umständen an redundanten Bildelementen erkannt werden.

Ein Beispiel: Bei einem iranischen Raketentest 2008 funktionierte eine von vier Raketen nicht. Um ein perfektes Bild vom Test zu transportieren, wurde der Fehler durch das Kopieren und Einfügen einer funktionierenden Rakete überdeckt. ${ }^{8}$

Die Strategie "Copy and Paste» kann innerhalb eines Fotos angewandt werden, aber auch im Zusammenhang mit mehreren Bildern. Es kommt auch vor, dass aus zwei ähnlichen Fotos ein einziges zusammengestellt wird. Teilweise ist dies da-

7 Vgl. http://www.rhetorik.ch/Aktuell/Aktuell_Feb_15_2003.html.

8 Für Abb. vgl. http://www.stern.de/politik/ausland/militaer-propaganda-iran-faelscht-fotos-vonraketentest-626808.html. 
durch zu erkennen, dass Bildelemente doppelt vorkommen ( $\mathrm{vgl}$. Ammann et al. 2012b, 59).

Folgende Veränderungen werden häufig im Rahmen digitaler Montage durchgeführt: Bildelemente entfernen, Bildelemente hinzufügen, Bildelemente verschiedener Fotos kombinieren, Objekte ausschneiden bzw. freistellen und in einen neuen Kontext einfügen.

\section{Bedeutungskonstruktion durch Präsentationskontexte}

Der unmittelbare Kontext, in dem eine fotografische Abbildung präsentiert wird, kann für die Bedeutungszuschreibung mitbestimmend sein. Ein und dasselbe Foto wird unterschiedlich wahrgenommen, je nachdem, wie es dem Betrachter vermittelt wird und in welchem Rahmen es gezeigt wird (im wörtlichen sowie im übertragenen Sinn). Folgende Fragen können dabei von Wichtigkeit sein: Wo wird das Bild aufbewahrt (Briefumschlag, Fotoalbum, Schuhkarton, Geldbeutel, externe Festplatte etc.)? Wo ist das Bild platziert (Schreibtisch, Zimmerwand, Plakatwand, Kunstausstellung, Museum, Internet-Galerie, Facebook, Flickr, Fotoblog etc.)? Wie wird das Bild präsentiert (Art der Rahmung, Art der Beleuchtung etc.)? Welcher Art ist das Medium, in dem das Bild abgebildet ist (Buch, Zeitung, Illustrierte, wissenschaftliche Zeitschrift, Werbeprospekt etc.)?

\section{Bedeutungskonstruktion durch Text}

Das geschriebene Wort stellt das Bild in einen erweiterten Bedeutungszusammenhang. Dazu schreibt Freund: «Die Objektivität des Bildes ist nur eine Illusion. Die Kommentare, die man ihm mitgibt, können seine Bedeutung völlig verändern» (Freund 1993, 172). In der künstlerischen Fotografie kann etwa eine bestimmte Bildunterschrift bzw. ein Titel die interpretatorische Offenheit einschränken und eine bestimmte Deutung nahe legen oder vorprägen. Je nach Fall oder Standpunkt kann eine solche Nachbarschaft von Bild und Wort als Zugangshilfe oder Interpretationseinengung aufgefasst werden. "Wir erleben es oft, dass sich mit der Änderung der beigefügten Worte der ganze Sinngehalt des Bildes zu ändern scheint und unter Umständen sogar in sein Gegenteil verkehrt» (Beiler 1977, 71). Diese Position verdeutlicht, dass durch nachträgliche Textzuordnung die ursprüngliche Intention der Fotografin bzw. des Fotografen in andere Bahnen umgelenkt werden kann.

In der Pressefotografie kann einem Bild durch Textunterschrift eine Bedeutung zugemessen werden, die weit über das Bild an sich hinausreicht, die aber gerade durch den Beweischarakter der Fotografie glaubhaft wird. Möglicherweise könnte also die Beweiskraft des Fotos auf Begleittexte überstrahlen und damit schriftliche Inhalte glaubhaft erscheinen lassen, die im Foto selbst nicht bewiesen sind. (Schuster 1996, 205). Eine Bildunterschrift kann dem Rezipienten auf der einen Sei- 
te helfen, die Abbildung in einem raum-zeitlichen Kontext zu verorten und damit die Herstellung einer Bedeutung zu erleichtern, auf der anderen Seite kann sie die Rezeption im Sinne einer verzerrenden Darstellung manipulieren.

Beispielhaft hierfür sei das Foto eines islamischen Geistlichen genannt, der - aus der Froschperspektive aufgenommen - mit erhobenen Armen vor einem brennenden Gebäude steht. Das Foto wurde dann in zwei verschiedenen Publikationskontexten mit zwei unterschiedlichen Bildunterschriften versehen:

A: «Ein islamischer Geistlicher versucht die Menge zu beschwichtigen.»

B: «Ein Geistlicher heizt die Stimmung aufgebrachter Gläubiger in der libanesischen Hauptstadt an.»

Das Beispiel macht deutlich, wie ein mehrdeutiges Foto durch Bildunterschriften in sehr entgegen gesetzte Richtungen gelenkt werden kann (Museum für Kommunikation, Bern \& Stiftung Haus der Geschichte der Bundesrepublik Deutschland 2007, 13).

\section{KARIKATURENSTREIT}

Diese Fotografie entstand in Beirut im Frühjahr 2006 im Zusammenhang mit den Protesten im so genannten «Karikaturenstreit». Es zeigt einen libanesischen Geistlichen vor dem brennenden dänischen Konsulat.

Beschreiben Sie die Rolle der Medien in diesem Konflikt.

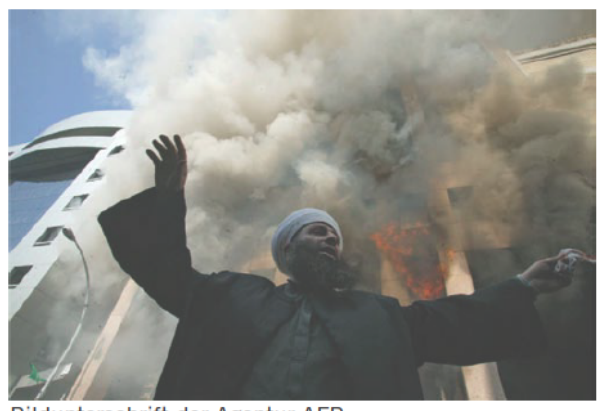

Bildunterschrift der Agentur AFP:

Ein islamischer Geistlicher versucht die Menge zu beschwichtigen.

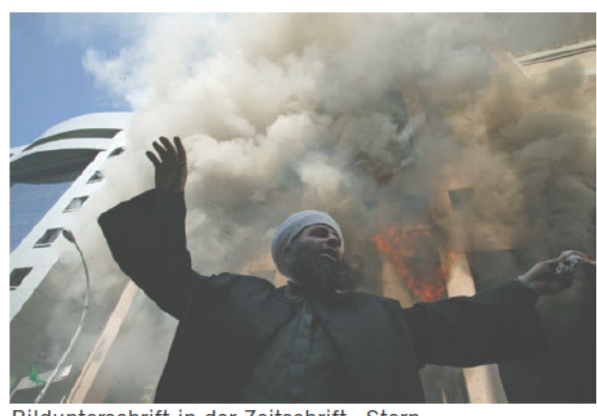

Ein Geistlicher heizt die Stimmung aufgebrachter Gläubiger in der libanesischen Hauptstadt an.

Abb. 19: Gleiches Bild - unterschiedliche Bildunterschriften 

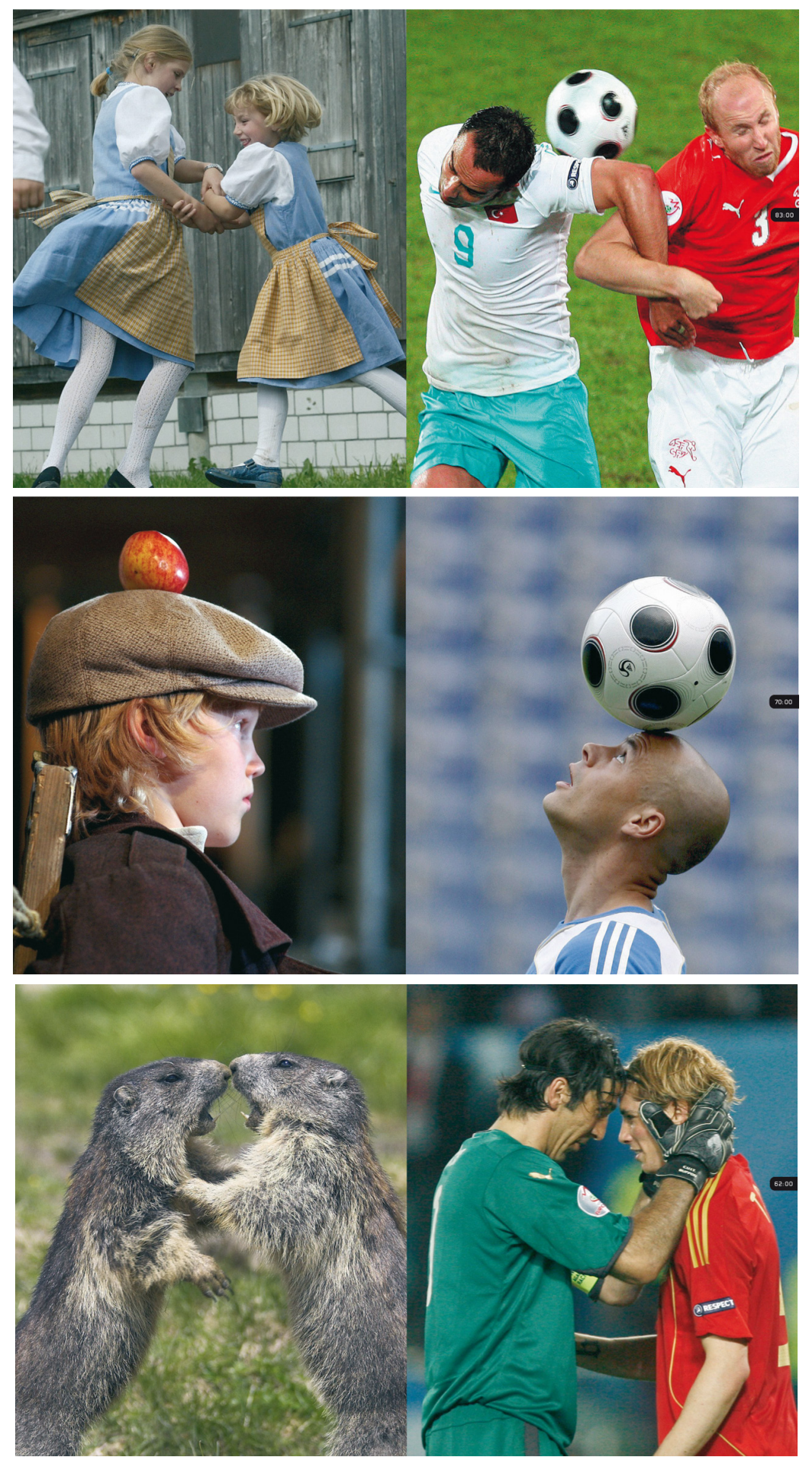

Abb. 20-22: Bildnachbarschaften aus Football - Switzerland 1:1 von Alain Picard 


\section{Bedeutungskonstruktion durch Nachbarschaft zu anderen Bildern}

Auch die Nachbarschaft eines Bildes mit einem anderen Bild oder mit andern Bildern kann eine Bedeutungsveränderung mit sich bringen. Bei der Erstellung von visuellen Kommunikationsträgern wie (Foto-)Kollagen oder Plakaten findet diese Eigenschaft eine gezielte Anwendung. John Heartfield hat sich diese Methode für seine politischen Fotokollagen zu Diensten gemacht (Freund 1993, 170).

Die Vieldeutigkeit eines Einzelbildes kann durch die Nachbarschaft zu anderen Bilden eingeschränkt (Günter 1977, 46) oder auch erweitert werden. Es besteht die Möglichkeit, Bildergeschichten zu erzählen (vgl. Duane Michals ${ }^{9}$ ) oder Veränderungen visuell darzustellen (etwa durch Aufnahmen desselben Ortes zu unterschiedlichen Zeitpunkten) (vgl. die Timescape-Projekte von Michael Ruetz ${ }^{10}$ ). Abbildungen aus unterschiedlichen Zeiten und von unterschiedlichen Orten können schliesslich gleichzeitig (und am selben Ort) dargeboten werden.

Durch die Nachbarschaft von Bildern lassen sich gestalterische (formale) und inhaltliche Kontraste erzeugen, wobei inhaltlicher und gestalterischer Kontrast auch Hand in Hand gehen können, um eine intendierte Wirkung zu erzeugen (z. B. Armut vs. Reichtum, Krieg vs. Frieden, Gewalt vs. Harmonie, Leben vs. Tod, Vergangenheit vs. Gegenwart, Alter vs. Jugend, Männlichkeit vs. Weiblichkeit, Schwarz vs. Weiss, Schwarzweiss vs. Farbe, Linie vs. Fläche, Dichte vs. Offenheit, Dynamik vs. Ruhe etc.).

Interessant ist in diesem Zusammenhang eine Arbeit von Alain Picard. In seinem Projekt Football - Switzerland 1:111 stellt er Bilder aus dem Kontext Fussball andern Bilder mit Bezug zur Schweiz gegenüber und zeigt damit sehr eindrücklich, wie durch Bildnachbarschaften neue Bedeutungspotenziale geschaffen werden können.

\section{Bedeutungskonstruktion durch akustisch-musikalischen Kontext}

Auch durch die Unterlegung eines Bildes mit bestimmten akustischen Reizen können Bedeutungen erzeugt werden, die dem Bild nicht inhärent sind. Im Alltagsleben findet sich diese Art der Bedeutungsveränderung vor allem bei bewegten Bildern (Kinofilme, Fernsehfilme und vor allem Werbespots), aber auch bei Formaten wie akustisch unterlegten Dia-Shows.

Ein Lufthansa-Werbefilm beispielsweise zeigt zweimal hintereinander die gleichen assoziativ geschnittenen Sequenzen von New York. Beim ersten Mal ist eine chaotische Musik unterlegt, beim zweiten Mal eine ruhige und harmonische. Je nach Musik wird das Filmmaterial anders wahrgenommen. Am Ende wird folgender

\footnotetext{
9 Vgl. http://duanemichals.tumblr.com/.

10 Vgl. http://www.michael-ruetz.de/index.php/2012-03-12-19-08-10/menu-options/2012-01-30-22-30-20.

11 Vgl. Projektorganisation Öffentliche Hand UEFA EURO 2008, 2008.
} 
Text eingeblendet: «You see the world the way you fly!» Dann folgt der Schriftzug "Lufthansa». ${ }^{12}$

\section{Bedeutungskonstruktion durch Herauslösung eines Einzelfotos}

Wird aus einer Filmsequenz ein Standbild herausgenommen, kann unter Umständen ein Bedeutungspotenzial entstehen, das im Bewegtbildablauf nicht gegeben gewesen wäre. Ein Beispiel ist das Obama-McCain TV Duell vom 15.10.2008. McCain streckt am Ende kurz die Zunge aus dem Mund. Als Einzelbild wirkt die Szene sehr irritierend, in der Filmsequenz jedoch nicht. ${ }^{13}$ Die Selektion von Standbildern aus einem Film weist durchaus Parallelen zum Fotografieren auf: Aus einer Fülle von möglichen Wirklichkeitsaspekten wird ein Aspekt herausgeholt und festgehalten. Bei der Auswahl eines Standbildes aus einem Film hat man jedoch die Möglichkeit, in Ruhe auszuwählen, das Material kann immer wieder betrachtet werden.

\section{Bilder ohne Wirklichkeitsreferenz}

Viele Bildmanipulationen basieren auf Fotografien, die im Nachhinein analog oder digital verändert wurden. Vorstellbar sind aber auch Bildprodukte, die gänzlich virtuell entstanden sind. Diese Bilder haben keine Wirklichkeitsreferenz im Sinne einer physikalischen Abbildung. Bekannt sind solche virtuellen Bildwelten beispielsweise aus Computerspielen. Es ist anzunehmen, dass die Unterscheidung zwischen realen Fotografien und virtuell generierten Bildern mit zunehmender technischer Perfektion immer schwieriger wird. In Anlehnung an Ridley Scotts Film «Blade Runner» könnte man von «fotografischen Replikanten» sprechen.

Im diesem Kontext wird auch von Computer-generated imagery (CGI) gesprochen.

\section{Pädagogische Kontexte}

Für pädagogische Kontexte ergeben sich vielfältige Möglichkeiten, das Phänomen Bildmanipulation zu thematisieren (vgl. Holzwarth 2011; Holzwarth 2012). Dabei können sowohl kognitiv orientierte (z. B. Diskussionen über Begriffsdefinitionen) als auch handlungsorientierte Zugänge gewählt werden (z. B. Bildausschnitte ergänzen oder Bedeutungsveränderungen durch Bildunterschriften). Ein wichtiger Aspekt kann darin bestehen, verschiedene Möglichkeiten der Bedeutungskonstruktion anhand von vorgefundenen oder selbst produzierten Beispielen bewusst zu machen und deren manipulatives Potenzial zu reflektieren. Ein differenzierter und kontextsensitiver Umgang mit dem Begriff «Manipulation» ist ein weiteres mögliches Lernziel.

Darüber hinaus stellt sich die Frage, inwieweit Fotografie als Mittel der Bereicherung und Veränderung von Sichtweisen nutzbar gemacht werden kann. Es kann

12 Vgl. http://youtu.be/VPjmJOzsP28.

13 Vgl. http://www.rhetorik.ch/Aktuell/08/10_15/index.html.

Peter Holzwarth www.medienpaed.com > 9.8.2013 
sich dabei sowohl um Bilder handeln, die von anderen Menschen gemacht wurden als auch um eigene Fotografien.

Tabelle 4 zeigt vier verschiedene Typen von Bildern, die jeweils mit unterschiedlichen Potenzialen verbunden sind: 1. Fotografische Selbstportraits, 2. Portraits von anderen, 3. selbstproduzierte Fotos und 4. Fotos, die weder Portraits der eigenen Person noch selbst gemachte Bilder sind.

Tabelle 4: Verschiedene Bildtypen als Ausgangspunkte für das Arbeiten mit Fotografie

\begin{tabular}{|l|l|l|}
\hline & Selbstproduzierte Fotos & Von anderen produzierte Fotos \\
\hline $\begin{array}{l}\text { Die eigene Per- } \\
\text { son als Gegen- } \\
\text { stand der Foto- } \\
\text { grafie }\end{array}$ & $\begin{array}{l}\text { 1) Fotografische Selbstportraits } \\
\text { (z. B. Spiegelbild fotografieren, sich } \\
\text { mit ausgestrecktem Arm selbst foto- } \\
\text { grafieren, sich mit dem Selbstauslöser } \\
\text { fotografieren) }\end{array}$ & 2) Portraits der eigenen Person \\
\hline $\begin{array}{l}\text { Andere Objekte } \\
\text { als Gegenstand } \\
\text { der Fotografie }\end{array}$ & $\begin{array}{l}\text { 3) Selbstproduzierte Fotos (z. B. das } \\
\text { eigene Zimmer / die eigene Wohnung } \\
\text { / das eigene Quartier) }\end{array}$ & $\begin{array}{l}\text { 4) Fotos, die weder Portraits der eigenen } \\
\text { Person noch selbst produzierte Bilder sind } \\
\text { (z. B. «Bilderserie So isst der Mensch»: Menzel } \\
\text { u. D'Aluisio 2005) }\end{array}$ \\
\hline
\end{tabular}

Das fotografische Abbild (vor allem Typ 4) kann Rezipierenden als Projektionsfläche dienen. Indem das Abgebildete aus seinem Kontext herausgelöst wird, entsteht eine Offenheit hinsichtlich der möglichen Bedeutungszuschreibungen. Bildbetrachtende können sich (ihre Erfahrungen, Gefühle, Erinnerungen etc.) mit der Fotografie in Beziehung bringen und dadurch solcher nach aussen verlagerten Aspekte bewusst werden. Der Schriftsteller Dieter Wellershoff hat das schön formuliert: «Wir treten durch Bilder mit uns selbst und der Welt in Beziehung» (Wellershoff 1980, 7). Das Bild wird gleichsam zum Anderen, in dem wir uns spiegeln. Dadurch, dass Betrachtende von sich etwas in das Bild «hineinsehen», können neue Sichtweisen auf die eigene Person entstehen. Rezipierende können sich möglicherweise über eine bestimmte Sache klarer werden. Durch die Verlagerung nach aussen kann sich das Subjekt zum Objekt machen.

Es ist anzunehmen, dass Rezipierende in einer ambivalenten Abbildung nur solche Dinge wahrnehmen können, die sie zuvor schon einmal gesehen oder erfahren haben. In diesem Sinne könnte Bildrezeption allgemein als ein Prozess der Projektion und Identifikation verstanden werden. Bildproduzierende abstrahieren die eigene Erfahrung und heben sie auf eine allgemeinere Zugänglichkeitsebene. Rezipierende können sich in den künstlerischen Ausdrucksformen spiegeln bzw. wiederfinden oder neuentdecken. 
Einen Sonderfall stellt die Auseinandersetzung mit dem eigenen Abbild dar (Typ 1 und 2): Im Rahmen des Forschungsprojekts «Fotografie und Selbstbild» (Gerig 2005) wurde der Zusammenhang von fotografischen Abbildungen von Personen und ihrem Selbstbild untersucht. Es ging um die Frage, ob Selbstbilder durch die Reflexion alter und neuer Fotos verändert werden können. Die Teilnehmenden konnten aus ihrem privaten Archiv 10 Fotos auswählen, über die im Rahmen eines Interviews gesprochen wurde. Das Projektteam wählte weitere Bilder für spezielle Bildkombinationen aus. Später wurden neue Bilder in einem Studiosetting und im öffentlichen Raum produziert. In diesem Zusammenhang ist auch das Konzept "photo therapy» zu nennen. ${ }^{14}$

Durch das Medium Fotografie kann das fotografierende Subjekt die eigene Lebenswelt aus neuer Perspektive erfahren (insbesondere Typ 1 und 3). Eingefahrene und erstarrte Sichtweisen (auch bezüglich der eigenen Person) können erfrischt und aufgelockert werden. Die fotografisch vermittelte Wirklichkeitsaneignung schafft eine Distanz zwischen Beobachter und Beobachtetem und eignet sich daher als Mittel der Bewusstmachung. Pädagogische Praxiskontexte können sich diese Eigenschaften zu Nutze machen:

Die Fotografie lässt sich durchaus als 〈Tool〉 zur Bewältigung des Alltags einsetzen. Beobachtung schafft Distanz. Gleichzeitig wird Anteilnahme möglich. Ein Bild entsteht, Erinnerungen werden festgehalten. Und wenn einzelne mit der Kamera in der Hand eine Beobachterposition einnehmen, bringt das auch Bewegung in festgefahrene Cliquenstrukturen. (Schober 1996, 75)

Über das Mittel der Fotografie kann der eigenen Persönlichkeit und dem jeweiligen Lebensgefühl Ausdruck verliehen werden. Im Gegenzug dienen den Rezipierenden fotografische Ausdrucksweisen als Verständnis- und Zugangshilfe für fremde Lebenswelten. Schuster hat darauf hingewiesen, dass Fotografien ausgezeichnet als Grundlage für Interviews genutzt werden können (vgl. Hurworth 2003):

Die Bilder erlauben im späteren Interview einen besseren Zugriff zu den Gefühlslagen und damit auch deren Bearbeitung. Verschiedene Randgruppen zeigen die Welt aus ihrem Blickwinkel, führen mit ihren Fotos zu den wichtigen Bildern ihres Lebens. Will man mit einem Interview etwas über das Leben z. B. von Obdachlosen erfahren, wird es wertvolle Informationen erbringen, einige Obdachlose mit einer Kamera auszustatten und sie zu bitten, Bilder von Objekten oder Personen anzufertigen, die in ihrem Leben etwas bedeuten. Ein späteres Interview zu diesen Inhalten geht gleich von einem

14 Vgl. http://www.phototherapy-centre.com/. 
Bezugssystem des Befragten aus und führt so leichter zu wesentlichen Informationen. (Schuster 1996, 83ff.)

Eine weitere Funktion kann darin bestehen, sich über die Fotografie der Relativität von Wirklichkeitsdeutungen und Wahrnehmungsstandpunkten bewusst zu werden.

Es ist deutlich geworden, dass Bildbedeutungen zu unterschiedlichen Zeitpunkten im Darstellungsprozess geprägt und verändert werden können. Dabei macht es Sinn, denn Begriff Bildmanipulation sehr differenziert zu gebrauchen und dabei nicht nur die Dimension der direkten mechanischen oder digitalen Veränderung am Bild einzubeziehen. Oft muss auch der genaue Kontext der Bildproduktion und der Bildrezeption betrachtet werden um entscheiden zu können, ob von Bildgestaltung oder Bildmanipulation gesprochen werden sollte. Für pädagogische Kontexte ergeben sich bei der Vermittlung visueller Kompetenz zahlreiche Herausforderungen aber auch viele spannende Möglichkeiten der lebensweltnahen Thematisierung.

\section{Literatur}

Ammann, Daniel, Urs Ingold, Flurin Senn, Silvie Spiess und Friederike Tilemann. 2012a. Medienkompass 1: Medien und ICT für die Primarstufe. 4., korr. Aufl. Zürich: Lehrmittelverlag.

Ammann, Daniel, Urs Ingold, Flurin Senn, Silvie Spiess und Friederike Tilemann. 2012b. Medienkompass 2: Medien und ICT für die Sekundarstufe. 3., korr. Aufl. Zürich: Lehrmittelverlag.

Barthes, Roland. 1985. Die helle Kammer. Bemerkungen zur Fotografie. Frankfurt am Main: Suhrkamp.

Beiler, Berthold. 1977. Weltanschauung der Fotografie. München: Damnitz.

Buddemeier, Heinz. 1981. Das Foto: Geschichte und Theorie der Fotografie als Grundlage eines neuen Urteils. Reinbek bei Hamburg: Rowohlt.

Bühl, Achim. 1986. Cybersociety: Mythos und Realität der Informationsgesellschaft. Köln: papyrossa.

Freund, Gisèle. 1993. Photographie und Gesellschaft. Hamburg: Rowohlt.

Gerig, Manfred 2005. Fotografie und Selbstbild: Personen sehen sich neu. Zürich: Hochschule für Gestaltung und Kunst.

Günter, Roland. 1977. Fotografie als Waffe: Geschichte der sozialdokumentarischen Fotografie. Hamburg: VSA-Verlag.

Haus der Geschichte der Bundesrepublik Deutschland, Hrsg. 2003. Bilder, die lügen. Bundeszentrale für politische Bildung. Bonn: Bouvier.

Hepp, Andreas. 1999. Cultural Studies und Medienanalyse. Opladen/Wiesbaden: Westdeutscher Verlag. 
Holzwarth, Peter. 2011. Kreative Medienarbeit mit Fotografie, Video und Audio: Grosse und kleine Projektideen für die medienpädagogische Praxis. München: kopaed.

Holzwarth, Peter. 2012. Menschen verändern Bilder - Bilder verändern Menschen. Dossiers Medien im Kontext. Zürich: Pädagogische Hochschule Zürich. http:// www.phzh.ch/Documents/phzh.ch/Medienbildung/Dokumente/Dossier_Bildmanipulation_2012-12.pdf (http://www.phzh.ch/de/medienbildung/Dossiers/).

Hurworth, Rosalind. 2003. Photo-Interviewing for research. Social Research UPDATE. University of Surrey. http://www.soc.surrey.ac.uk/sru/SRU40.pdf.

Kerouac, Jack. (1957) 1991. On the Road. London: Penguin Books.

Menzel, Peter und Faith D'Aluisio. 2005. So isst der Mensch. Familien in aller Welt zeigen, was sie ernährt. Hamburg: Gruner \& Jahr.

Museum für Kommunikation, Bern; Stiftung Haus der Geschichte der Bundesrepublik Deutschland. 2007. Bilder, die lügen - didaktische Materialien. http:// www.mfk.ch/fileadmin/pdfs/Bildung_Vermittlung/Materialien/Ausstellungen/ Bdl/Bdl_didakt_Materialien.pdf.

Orwell, George. 1990. 1984. Frankfurt am Main: Ullstein.

Projektorganisation Öffentliche Hand UEFA EURO 2008, Hrsg. 2008. Football Switzerland 1:1. Typo Ankick.

Sander, Uwe, Friederike von Gross und Kai-Uwe Hugger, Hrsg. 2008. Handbuch Medienpädagogik. Wiesbaden: VS Verlag für Sozialwissenschaften.

Schneider, Birgit. 1993. "Reisefotografie.» In Tourismuspsychologie und Tourismussoziologie: Ein Handbuch zur Tourismuswissenschaft, hrsg. v. Heinz Hahn u. Jürgen H. Kagelmann, S. 447-454. München: Quintessenz.

Schober, Barbara, Silke und Harten-Preiss, Hrsg. 1996. Wir schiessen zurück: Fotografie als GegenKULTURgut. Münster: Agenda.

Schuster, Martin. 1996. Fotopsychologie: Lächeln für die Ewigkeit. Berlin, Heidelberg: Springer.

Sontag, Susan. 1995. Über Fotografie. Frankfurt am Main: Fischer.

Stiegler, Bernd. 2009. Montagen des Realen: Photographie als Reflexionsmedium und Kulturtechnik. München: Fink.

Watterson, Bill. 1993. The Days Are Just Packed: A Calvin and Hobbes Collection by Bill Watterson. Kansas City.

Wellershoff, Dieter. 1980. «Inszenierte Wahrnehmung. Zu den Bildern dieser Ausstellung.» In Vorstellungen und Wirklichkeit - 7 Aspekte subjektiver Fotografie, hrsg. v. Rolf Wedewer, S. 7-21. Köln: Wienand.

[letzter Zugriff auf alle Webseiten im Beitrag am 9.1.2013]

Peter Holzwarth www.medienpaed.com > 9.8.2013 


\section{Abbildungsverzeichnis}

Abb. 1: Archiv Peter Holzwarth

Abb. 2: Haus der Geschichte der Bundesrepublik Deutschland 2003, 84

http://en.wikipedia.org/wiki/Photo_manipulation

Abb. 3: Sebastian Niedlich

http://www.flickr.com/photos/42311564@N00/494023970/in/set-

72157601948950307

Abb. 4: http://www.lsg.musin.de/geschichte/Material/referate/lkg/

krimkrieg_1856.htm

Abb. 5: Peter Holzwarth

Abb. 6-9: Peter Holzwarth

Abb. 10-13: Peter Holzwarth

Abb. 14: Peter Holzwarth

Abb. 15: Peter Holzwarth

Abb. 16 und 17: Peter Holzwarth

Abb. 18: Ursula Dahmen, Museum für Kommunikation Bern

http://www.mfk.ch/fileadmin/mfkpics/medienbilder/Bilderluegen/

IRAKISCHER_SOLDAT2.tif

Abb. 19: Museum für Kommunikation \& Haus der Geschichte der Bundesrepublik

Deutschland 2007, S. 13

Abb. 20-22: Alain Picard, http://www.footballswitzerland.ch/ 